\title{
Changes in Selected Brain Neurotransmitters and Their Metabolites in the Lamb after Thyroidectomy during the Last Two Trimesters of Gestation or the Early Neonatal Period
}

\author{
GAIL E. RICHARDS, PETER D. GLUCKMAN, KAREN BALL, SANDRA C. MANNELLI, AND \\ JULIE A. KALAMARAS \\ Department of Pediatrics, Northwestern University Medical School and Children's Memorial Hospital, \\ Chicago, Illinois 60614 and Developmental Physiology Laboratory, Department of Pediatrics \\ University of Auckland, Auckland, New Zealand
}

\begin{abstract}
To evaluate in a developmental context the effect of congenital hypothyroidism on concentrations of the neurotransmitters norepinephrine, dopamine, and serotonin (5HT) in selected brain areas of the ovine fetus, we studied the effect of thyroidectomy at three ages on the concentrations of these neurotransmitters and their major metabolites, homovanillic acid and 5-hydroxyindoleacetic acid. Fetuses underwent thyroidectomy at $90-95$ or $105-$

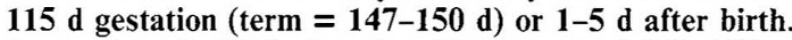
Approximately $25 \mathrm{~d}$ after thyroidectomy, at d 120-125 or 130-135 of gestation or 25-30 d after birth, respectively, the ewes were killed and fetal brains removed. Neurotransmitters and their metabolites were measured by HPLC with electrochemical detection. Thyroidectomy in the 2nd trimester increased 5HT in five brain areas: anterior hypothalamus, dorsal medial hypothalamus, pons, medulla, and cerebellum. Thyroidectomy in the 3rd trimester increased $5 \mathrm{HT}$ in the pons and medulla, increased norepinephrine in the dorsal medial hypothalamus and pons, and increased homovanillic acid in the posterior hypothalamus. Thyroidectomy in the newborn period decreased NE in the anterior hypothalamus, ventral medial hypothalamus, and midbrain, decreased 5-hydroxyindoleactic acid in the posterior hypothalamus, lateral hypothalamus, dorsal medial hypothalamus, and ventral medial hypothalamus, and decreased homovanillic acid in the dorsal medial hypothalamus and ventral medial hypothalamus. From these data we conclude the following: 1 ) Hypothyroidism causes changes in neurotransmitter concentrations only in selected brain areas of the ovine fetus, rather than causing generalized and similar changes in all brain ares; and 2) 5HT 5hydroxyindoleacetic acid concentrations are affected more often than the other neurotransmitters evaluated, perhaps because the $5 \mathrm{HT}$ neurotransmitter system is developing at these times. (Pediatr Res 28: 469-472, 1990)
\end{abstract}

\section{Abbreviations}

AH, anterior hypothalamus

DMH, dorsomedial hypothalamus

LaH, lateral hypothalamus

$\mathrm{PH}$, posterior hypothalamus

Received February 19. 1990: accepted June 5, 1990

Correspondence and reprint requests: Gail E. Richards, M.D., Division of Endocrinology, Children's Memorial Hospital, 2300 Children's Plaza, Chicago, IL 60614

Supported in part by Grant HD 21075 from the National Institutes of Health and grants from The Medical Research Council of New Zealand and the Wellcome Trust.
VMH, ventromedial hypothalamus

NE, norepinephrine

DA, dopamine

HVA, homovanillic acid

5HT, serotonin

HIAA, 5-hydroxyindoleacetic acid

NT, neurotransmitter

Tx2, thyroidectomy in the 2nd trimester

Tx3, thyroidectomy in the 3rd trimester

TxNB, thyroidectomy in the newborn period

Hypothyroidism has been shown to affect most aspects of brain development including DNA content, protein content, NT content, synaptic organization, and myelination (1-3). Defining the mechanism(s) that causes these effects has proven difficult, however. The effects of hypothyroidism have been described in several species in relation to gestational age rather than a physiologic maturational event, and species differ in the time of maturational events in the brain.

Available data from various mammalian species concerning the effect of fetal hypothyroidism on brain NT are not consistent and do not give a clear picture of the mechanism by which brain (or brain nuclei) concentrations of the various NT are affected by hypothyroidism (4-10). We have chosen to study an ovine model because of the large size of the fetus and its accessibility for in utero thyroidectomy as well as the large body of data indicating similarity between human and ovine fetal thyroid physiology (11). We have therefore designed experiments to test the following hypothesis: Thyroidectomy in the ovine fetus causes distinctive changes in brain NT concentrations that are dependent on the developmental state of the animal at the time of thyroidectomy. Data for the control animals have also been reported elsewhere (Richards GE, Gluckman PD, Ball K, Mannelli SC, Kalamaras J, unpublished observations).

\section{MATERIALS AND METHODS}

Animals. Romney ewes were mated to Suffolk rams on a known date to produce fetuses of known gestational age for our study. Pregnant ewes were brought from the breeding farm to the Developmental Physiology Laboratory, University of Auckland, a few days before surgery. All procedures were approved by the Animal Ethical Committee of the University of Auckland.

Surgery. After an overnight fast, the animals were anesthetized with i.v. aphaxalone and maintained with halothane/oxygen via 
an endotracheal tube. Using sterile technique, the fetal head was delivered through a midline abdominal incision. The fetal thyroid was removed, the fetus was returned to the uterus, and the uterus and abdomen were closed. Newborn animals had thyroidectomies performed after a 6-h fast under halothane/oxygen anesthesia.

Protocol. Postoperatively, fetuses were allowed to develop undisturbed until they were killed. Newborns were returned to their mothers and allowed to grow without further intervention until they were killed. Each group of animals was hypothyroid for approximately $25 \mathrm{~d}$, after which the ewe or newborn was killed by barbiturate overdose and the fetal or newborn brain removed and frozen at $-80^{\circ} \mathrm{C}$ within $10-15$ min of death.

Experimental groups. Tx2 $(n=5)$ animals underwent thyroidectomy at 90-95 d gestation and were killed at 120-125 d gestation. Tx3 $(n=10)$ animals underwent thyroidectomy at $105-115 \mathrm{~d}$ gestation and were killed at 133-137 d gestation. TxNB $(n=5)$ animals underwent thyroidectomy at $1-5 \mathrm{~d}$ after birth and were killed at 25-30 d of age.

Plasma thyroxine was less than $25 \mathrm{nmol} / \mathrm{L}$ in every thyroidectomized animal at the time of death. In another group of animals $(n=6)$ not included in this report, we have verified that plasma thyroxine falls to less than $25 \mathrm{nmol}$ by $48 \mathrm{~h}$ after surgery. Thus, we are confident of the completeness of thyroidectomy and the period of time during which the animals were hypothyroid.

Controls. Control animals were either unoperated twins of thyroidectomy animals, or animals who had carotid artery and jugular vein catheter placement without thyroidectomy and without any other experimental intervention. The procedure for killing the ewe and collecting the fetal brains was the same in all groups. There were five control animals for the Tx2 group (120125 d gestation), eight control animals for the Tx3 group (133$137 \mathrm{~d}$ gestation), and five control animals for the TxNB group (25-30 d of age). Plasma thyroxine at the time of death in control animals was $154 \pm 10.9 \mathrm{nmol} / \mathrm{L}$, a value comparable to that reported by others (11-13).

NT. NT and metabolites were measured by HPLC with electrochemical detection as previously described (14). This method quantifies NE, DA and its major metabolite HVA, and 5HT and its major metabolite HIAA. 3-Methoxy-4-hydroxyphenylethylene glycol, the major metabolite of NE, could be measured on some occasions, but the peak merged with the solvent front often enough that 3-methoxy-4-hydroxyphenylethylene glycol results were not reliable enough to report here. Brains were dissected while frozen into the following areas according to the atlas of Gluckman and Parsons (15): AH, PH, LaH, DMH, VMH, midbrain, pons, medulla, cerebrum, and cerebellum. Brain areas were never thawed before assay.

Thyroxine. Thyroxine was measured by RIA (16).

Statistics. Each thyroidectomy group was compared with its appropriate control group by unpai, ed two-tailed $t$ test (17). Differences are considered to be significant when $p<0.05$.

\section{RESULTS}

The effects of thyroidectomy in the developing sheep at three different ages are described in Tables 1-5. Of all the brain areas examined in the three thyroidectomy groups, $14 \%$ were significantly different from controls. Animals in the Tx2 group had no change in NE, DA, HVA, or HIAA, but 5 HT was significantly increased in five brain areas $\mathrm{AH}, \mathrm{DMH}$, pons, medulla, and cerebellum) when compared with controls. The Tx3 group had increased $\mathrm{NE}$ in the $\mathrm{AH}, \mathrm{DMH}$, and pons, no change in DA, increased HVA in the PH, increased 5HT in pons and medulla, and decreased HIAA in midbrain. When thyroidectomy was performed after birth (TxNB group), NE was decreased in the $\mathrm{AH}, \mathrm{VMH}$, and midbrain; DA was unchanged; HVA was decreased in the DMH and VMH; 5HT was unchanged; and HIAA decreased in four areas (PH, LaH, DMH, and VMH).
Of the 21 reported differences between controls and thyroidectomy animals, the majority (13 of 21 ) were observed in the 5 HT/HIAA system. To view these changes in another perspective, $5 \mathrm{HT}$ was increased in $50 \%$ of the brain areas in the Tx2 group and $20 \%$ of the Tx3 group. HIAA, on the other hand, was increased in only $10 \%$ of the Tx 2 group and $10 \%$ of the Tx3 group, but was decreased in $40 \%$ of the TxNB group. Thus, although the total number of changes was relatively small, the relative percentage of the brain affected was much larger for the 5HT/HIAA NT system.

\section{DISCUSSION}

We have demonstrated that the effects of thyroidectomy on NT concentration are not global changes that affect every brain area and every NT in the same manner throughout development. On the contrary, the effect of hypothyroidism is specific for brain area and NT, making it unlikely that these effects are simply the manifestation of decreased NT metabolism in all cells. Our findings expand the results of a study by Rastogi and Singhal (6) in a rat model, in which 5HT decreased and HIAA increased in several brain areas after ${ }^{131} \mathrm{I}$ administration on the day of birth, and the findings of Savard et al. (5) in rats treated with propylthiouracil for the first $42 \mathrm{~d}$ of life. In the latter study, the 5HT content of discrete nuclei rather than of larger brain areas was measured. 5HT was increased in 11 nuclei of the hypothyroid rats and HIAA was higher in 16 nuclei compared with controls. In a study of 100-d-old rats that had undergone surgical thyroidectomy 3 wk earlier, a decrease in 5HT accompanied by a decrease in DA and no change in NE was reported by Ito et al. (9). A decrease in NE with no change in DA or 5HT has also been demonstrated in rats given propylthiouracil for 15 or $30 \mathrm{~d}$ after birth (4). The same authors (8) also found decreased NE and DA in brain areas of rats given ${ }^{131} \mathrm{I}$ on the day of birth.

These cross-species comparisons are difficult to interpret and relate to hypothyroidism in humans because of differences in developmental timing in the brain with respect to birth: the rat undergoes relatively more postnatal differentiation than the human, whereas the sheep undergoes relatively more development in the prenatal period $(2,18)$. The choice of timing of thyroidectomy in our study was based on a heuristic assumption that the early part of the 3rd trimester in the sheep and early postnatal life in the human represent roughly equivalent stages of brain development. We chose for comparison a time before and after the period of maximal interest. In the hypothyroid neonatal rat, the reversibility of the changes in NT concentrations demonstrated after treatment beginning at postnatal $\mathrm{d} 5$ but not at postnatal d 20 (8) suggests that in this species, the critical time period for thyroxine's effect on NT lies somewhere between $\mathrm{d} 5$ and 20. Our studies cannot address at this time the issue of whether the changes in NT that we describe are reversible with thyroxine therapy.

There are a number of possible explanations for our findings. One hypothesis is that hypothyroidism produces a delay in the normal ontogeny of neural development by interfering with NT synthesis, which would be reflected in reduced concentrations of NT in certain brain areas. The increases in 5HT we observed in the Tx 2 and Tx 3 groups and in NE in the Tx 3 group are difficult to reconcile with this hypothesis. An alternative hypothesis is that hypothyroidism affects NT concentrations by interfering with normal NT metabolism, rather than synthesis.

Increases in NT in the Tx2 and Tx3 groups are more compatible with this hypothesis, but the decreases observed in both NT and metabolites in the TxNB group do not easily fit a mechanism of action based solely on changes in the metabolic fate of a normally manufactured NT. Because neuronal ontogeny includes the development of the capacity both to synthesize and metabolize NT and these processes are usually closely synchronized, it is difficult to distinguish experimentally between these hypothetical effects of hypothyroidism. The difference in the 
NEUROTRANSMITTERS IN HYPOTHYROIDISM

Table 1. NE concentrations in $n g / m g$ (mean \pm SEM) in three groups of sheep undergoing thyroidectomy at different ages compared with controls*

\begin{tabular}{|c|c|c|c|c|c|c|}
\hline & Control & $T \times 2$ & Control & $\mathrm{T} \times 3$ & Control & TxNB \\
\hline $\mathrm{AH}$ & $0.57 \pm 0.21$ & $0.47 \pm 0.05$ & $0.50 \pm 0.08$ & $0.79 \pm 0.15$ & $1.31 \pm 0.18$ & $0.68 \pm 0.10 \dagger$ \\
\hline $\mathrm{PH}$ & $0.35 \pm 0.07$ & $0.31 \pm 0.06$ & $0.26 \pm 0.01$ & $0.31 \pm 0.02$ & $0.53 \pm 0.15$ & $0.44 \pm 0.05$ \\
\hline $\mathrm{LaH}$ & $0.60 \pm 0.28$ & $0.32 \pm 0.03$ & $0.40 \pm 0.09$ & $0.47 \pm 0.09$ & $0.91 \pm 0.11$ & $0.56 \pm 0.11$ \\
\hline DMH & $0.42 \pm 0.11$ & $0.53 \pm 0.03$ & $0.39 \pm 0.01$ & $0.80 \pm 0.15 \dagger$ & $1.40 \pm 0.23$ & $0.73 \pm 0.08$ \\
\hline VMH & $0.51 \pm 0.17$ & $0.45 \pm 0.06$ & $0.54 \pm 0.10$ & $0.56 \pm 0.09$ & $1.40 \pm 0.14$ & $0.89 \pm 0.18 \dagger$ \\
\hline Midbrain & $0.25 \pm 0.01$ & $0.34 \pm 0.05$ & $0.31 \pm 0.03$ & $0.31 \pm 0.02$ & $0.76 \pm 0.13$ & $0.37 \pm 0.02 \dagger$ \\
\hline Pons & $0.38 \pm 0.07$ & $0.47 \pm 0.07$ & $0.31 \pm 0.04$ & $0.50 \pm 0.07 \dagger$ & $0.43 \pm 0.06$ & $0.42 \pm 0.04$ \\
\hline Medulla & $0.41 \pm 0.12$ & $0.41 \pm 0.04$ & $0.29 \pm 0.02$ & $0.51 \pm 0.12$ & $0.52 \pm 0.09$ & $0.36 \pm 0.04$ \\
\hline Cerebrum & $0.25 \pm 0.01$ & $0.25 \pm 0.01$ & $0.27 \pm 0.02$ & $0.25 \pm 0.01$ & $0.26 \pm 0.01$ & $0.29 \pm 0.01$ \\
\hline Cerebellum & $0.25 \pm 0.01$ & $0.25 \pm 0.01$ & $0.26 \pm 0.01$ & $0.25 \pm 0.01$ & $0.30 \pm 0.02$ & $0.28 \pm 0.01$ \\
\hline
\end{tabular}

* Treatment and control groups are described in detail in Materials and Methods.

$+p<0.05$.

Table 2. DA concentrations in $n g / m g$ (mean \pm SEM) in three groups of sheep undergoing thyroidectomy at different ages compared with controls*

\begin{tabular}{|c|c|c|c|c|c|c|}
\hline & Control & $\mathrm{T} \times 2$ & Control & $\mathrm{T} \times 3$ & Control & TxNB \\
\hline $\mathrm{AH}$ & $0.25 \pm 0.01$ & $0.25 \pm 0.01$ & $0.25 \pm 0.01$ & $0.32 \pm 0.04$ & $0.26 \pm 0.02$ & $0.26 \pm 0.02$ \\
\hline $\mathrm{PH}$ & $0.28 \pm 0.03$ & $0.27 \pm 0.02$ & $0.25 \pm 0.01$ & $0.40 \pm 0.15$ & $0.26 \pm 0.01$ & $0.25 \pm 0.01$ \\
\hline $\mathrm{LaH}$ & $0.25 \pm 0.01$ & $0.35 \pm 0.07$ & $0.25 \pm 0.01$ & $0.28 \pm 0.04$ & $0.29 \pm 0.03$ & $0.26 \pm 0.01$ \\
\hline DMH & $0.25 \pm 0.01$ & $0.43 \pm 0.11$ & $0.27 \pm 0.02$ & $0.27 \pm 0.02$ & $0.27 \pm 0.01$ & $0.26 \pm 0.01$ \\
\hline VMH & $0.26 \pm 0.01$ & $0.42 \pm 0.12$ & $0.25 \pm 0.01$ & $0.28 \pm 0.03$ & $0.31 \pm 0.02$ & $0.26 \pm 0.01$ \\
\hline Midbrain & $0.26 \pm 0.01$ & $0.41 \pm 0.07$ & $0.27 \pm 0.02$ & $0.29 \pm 0.04$ & $0.29 \pm 0.03$ & $0.25 \pm 0.01$ \\
\hline Pons & $0.25 \pm 0.01$ & $0.36 \pm 0.07$ & $0.25 \pm 0.01$ & $0.31 \pm 0.03$ & $0.26 \pm 0.01$ & $0.26 \pm 0.01$ \\
\hline Medulla & $0.29 \pm 0.04$ & $0.36 \pm 0.07$ & $0.31 \pm 0.06$ & $0.28 \pm 0.03$ & $0.29 \pm 0.01$ & $0.25 \pm 0.01$ \\
\hline Cerebrum & $0.25 \pm 0.01$ & $0.38 \pm 0.08$ & $0.25 \pm 0.01$ & $0.27 \pm 0.02$ & $0.25 \pm 0.01$ & $0.25 \pm 0.01$ \\
\hline Cerebellum & $0.25 \pm 0.01$ & $0.36 \pm 0.07$ & $0.25 \pm 0.01$ & $0.44 \pm 0.18$ & $0.25 \pm 0.01$ & $0.25 \pm 0.01$ \\
\hline
\end{tabular}

* Treatment and control groups are described in detail in Materials and Methods.

Table 3. HVA concentrations in $n g / m g$ (mean \pm SEM) in three groups of sheep undergoing thyroidectomy at different ages compared with controls*

\begin{tabular}{|c|c|c|c|c|c|c|}
\hline & Control & $\mathrm{T} \times 2$ & Control & Tx3 & Control & TxNB \\
\hline $\mathrm{AH}$ & $0.51 \pm 0.13$ & $0.47 \pm 0.11$ & $0.55 \pm 0.12$ & $0.98 \pm 0.18$ & $0.64 \pm 0.12$ & $0.38 \pm 0.06$ \\
\hline $\mathrm{PH}$ & $0.39 \pm 0.05$ & $0.43 \pm 0.09$ & $0.28 \pm 0.01$ & $0.73 \pm 0.14 \dagger$ & $0.51 \pm 0.06$ & $0.34 \pm 0.04$ \\
\hline $\mathrm{LaH}$ & $0.49 \pm 0.10$ & $0.50 \pm 0.03$ & $0.56 \pm 0.01$ & $1.03 \pm 0.30$ & $0.67 \pm 0.10$ & $0.39 \pm 0.05$ \\
\hline $\mathrm{DMH}$ & $0.59 \pm 0.15$ & $0.54 \pm 0.14$ & $0.55 \pm 0.09$ & $0.81 \pm 0.16$ & $0.82 \pm 0.07$ & $0.37 \pm 0.04 \dagger$ \\
\hline $\mathrm{VMH}$ & $0.51 \pm 0.13$ & $0.36 \pm 0.07$ & $0.55 \pm 0.12$ & $0.77 \pm 0.21$ & $0.69 \pm 0.07$ & $0.36 \pm 0.04 \dagger$ \\
\hline Midbrain & $0.88 \pm 0.19$ & $0.62 \pm 0.18$ & $0.94 \pm 0.03$ & $0.90 \pm 0.16$ & $0.73 \pm 0.15$ & $0.55 \pm 0.05$ \\
\hline Pons & $0.48 \pm 0.11$ & $0.36 \pm 0.06$ & $0.64 \pm 0.19$ & $0.70 \pm 0.14$ & $0.29 \pm 0.12$ & $0.39 \pm 0.02$ \\
\hline Medulla & $0.47 \pm 0.11$ & $0.34 \pm 0.10$ & $0.32 \pm 0.04$ & $0.33 \pm 0.03$ & $0.31 \pm 0.05$ & $0.25 \pm 0.01$ \\
\hline Cerebrum & $0.51 \pm 0.14$ & $0.25 \pm 0.01$ & $0.31 \pm 0.05$ & $0.26 \pm 0.01$ & $0.26 \pm 0.01$ & $0.25 \pm 0.01$ \\
\hline Cerebellum & $0.41 \pm 0.10$ & $0.27 \pm 0.02$ & $0.26 \pm 0.01$ & $0.32 \pm 0.05$ & $0.25 \pm 0.01$ & $0.26 \pm 0.01$ \\
\hline
\end{tabular}

* Treatment and control groups are described in detail in Materials and Methods.

$+p<0.05$.

Table 4. 5HT concentrations in $n g / m g$ (mean \pm SEM) in three groups of sheep undergoing thyroidectomy at different ages compared with controls*

\begin{tabular}{|c|c|c|c|c|c|c|}
\hline & Control & $\mathrm{T} \times 2$ & Control & $\mathrm{T} \times 3$ & Control & TxNB \\
\hline $\mathrm{AH}$ & $0.25 \pm 0.01$ & $0.51 \pm 0.09 \dagger$ & $0.35 \pm 0.03$ & $0.59 \pm 0.17$ & $0.51 \pm 0.10$ & $0.32 \pm 0.04$ \\
\hline $\mathrm{PH}$ & $0.34 \pm 0.06$ & $0.53 \pm 0.07$ & $0.44 \pm 0.07$ & $0.53 \pm 0.12$ & $0.51 \pm 0.01$ & $0.32 \pm 0.03$ \\
\hline $\mathrm{LaH}$ & $0.89 \pm 0.32$ & $0.52 \pm 0.04$ & $0.45 \pm 0.11$ & $0.64 \pm 0.13$ & $0.65 \pm 0.23$ & $0.37 \pm 0.04$ \\
\hline $\mathrm{DMH}$ & $0.32 \pm 0.06$ & $0.69 \pm 0.06 \dagger$ & $0.53 \pm 0.18$ & $0.47 \pm 0.08$ & $0.63 \pm 0.16$ & $0.29 \pm 0.01$ \\
\hline VMH & $0.42 \pm 0.08$ & $0.54 \pm 0.07$ & $0.38 \pm 0.07$ & $0.58 \pm 0.11$ & $0.82 \pm 0.23$ & $0.34 \pm 0.04$ \\
\hline Midbrain & $0.94 \pm 0.37$ & $1.16 \pm 0.16$ & $0.68 \pm 0.16$ & $0.75 \pm 0.11$ & $0.84 \pm 0.24$ & $0.43 \pm 0.05$ \\
\hline Pons & $0.51 \pm 0.11$ & $0.93 \pm 0.03 \dagger$ & $0.40 \pm 0.05$ & $1.24 \pm 0.17 \dagger$ & $0.61 \pm 0.21$ & $0.41 \pm 0.03$ \\
\hline Medulla & $0.68 \pm 0.12$ & $1.10 \pm 0.05 t$ & $0.43 \pm 0.07$ & $0.88 \pm 0.16 \dagger$ & $0.64 \pm 0.21$ & $0.32 \pm 0.01$ \\
\hline Cerebrum & $0.29 \pm 0.03$ & $0.32 \pm 0.05$ & $0.33 \pm 0.06$ & $0.32 \pm 0.05$ & $0.62 \pm 0.26$ & $0.31 \pm 0.02$ \\
\hline Cerebellum & $0.45 \pm 0.11$ & $1.03 \pm 0.17 \dagger$ & $0.59 \pm 0.27$ & $0.65 \pm 0.18$ & $0.42 \pm 0.07$ & $0.26 \pm 0.01$ \\
\hline
\end{tabular}

$*$ Treatment and control groups are described in detail in Materials and Methods.

$+p<0.05$. 
Table 5. HIAA concentrations in $n g / m g$ (mean \pm SEM) in three groups of sheep undergoing thyroidectomy at different ages compared with controls*

\begin{tabular}{|c|c|c|c|c|c|c|}
\hline & Control & $\mathrm{T} \times 2$ & Control & $\mathrm{T} \times 3$ & Control & TxNB \\
\hline $\mathrm{AH}$ & $0.51 \pm 0.13$ & $0.56 \pm 0.10$ & $0.73 \pm 0.11$ & $0.77 \pm 0.10$ & $0.71 \pm 0.14$ & $0.50 \pm 0.11$ \\
\hline PH & $0.64 \pm 0.24$ & $0.60 \pm 0.09$ & $0.66 \pm 0.09$ & $0.71 \pm 0.09$ & $0.77 \pm 0.12$ & $0.36 \pm 0.03 \dagger$ \\
\hline $\mathrm{LaH}$ & $0.58 \pm 0.10$ & $0.53 \pm 0.06$ & $0.79 \pm 0.10$ & $1.03 \pm 0.15$ & $0.79 \pm 0.09$ & $0.40 \pm 0.08 \dagger$ \\
\hline $\mathrm{DMH}$ & $0.62 \pm 0.16$ & $0.70 \pm 0.06$ & $0.70 \pm 0.12$ & $0.79 \pm 0.09$ & $1.13 \pm 0.11$ & $0.40 \pm 0.03 \dagger$ \\
\hline $\mathrm{VMH}$ & $0.67 \pm 0.24$ & $0.49 \pm 0.05$ & $0.78 \pm 0.10$ & $0.79 \pm 0.08$ & $1.04 \pm 0.05$ & $0.49 \pm 0.05 \dagger$ \\
\hline Midbrain & $1.19 \pm 0.59$ & $1.07 \pm 0.22$ & $1.60 \pm 0.31$ & $0.93 \pm 0.08 \dagger$ & $1.35 \pm 0.38$ & $0.96 \pm 0.14$ \\
\hline Pons & $0.86 \pm 0.11$ & $1.94 \pm 0.19 \dagger$ & $1.49 \pm 0.21$ & $1.33 \pm 0.20$ & $1.34 \pm 0.70$ & $0.76 \pm 0.09$ \\
\hline Medulla & $1.23 \pm 0.31$ & $1.43 \pm 0.17$ & $0.91 \pm 0.20$ & $0.80 \pm 0.07$ & $1.14 \pm 0.18$ & $0.78 \pm 0.21$ \\
\hline Cerebrum & $0.59 \pm 0.16$ & $0.25 \pm 0.01$ & $0.41 \pm 0.08$ & $0.36 \pm 0.05$ & $0.42 \pm 0.07$ & $0.31 \pm 0.02$ \\
\hline Cerebellum & $0.55 \pm 0.18$ & $0.36 \pm 0.11$ & $0.35 \pm 0.06$ & $0.60 \pm 0.13$ & $0.37 \pm 0.06$ & $0.35 \pm 0.04$ \\
\hline
\end{tabular}

* Treatment and control groups are described in detail in Materials and Methods.

$\dagger p<0.05$.

direction of change of NT concentration when thyroidectomy occurs before versus after birth suggests that the mechanisms underlying these changes may be different at these two times.

Our study did not attempt to identify the specific mechanism by which thyroidectomy led to changes in NT concentrations, and our results could be interpreted as consistent with either hypothesis. In the lamb undergoing normal ontogeny, 5HT concentrations in early gestation have not been evaluated, but they remain stable while HIAA concentrations increase from $120-125 \mathrm{~d}$ through the newborn period (14; Richards GE, Gluckman PD, Ball K, Mannelli SC, Kalamaras J, unpublished observations). Our observation that HIAA concentrations decrease during this same period in the thyroidectomized fetus could be interpreted to suggest that the ontogeny of $5 \mathrm{HT}$ synthesis is arrested as a consequence of hypothyroidism.

In summary, our studies have demonstrated that in our fetal ovine model, hypothyroidism changes NT in several brain areas and the direction of these changes is consistent in its dependence on the stage of development of the animal. The specificity of these changes with respect to brain area and NT involved indicates that detailed studies of the synthesis and turnover of multiple NT will be necessary to clarify the mechanism of the changes we have observed.

\section{REFERENCES}

1. Nunez J 1984 Effect of thyroid hormones during brain differentiation. Mol Cell Endocrinol 37:125-132

2. Hetzel BS. Hay ID 1979 Thyroid function, iodine nutrition, and fetal brain development. Clin Endocrinol (Oxf) 11:445-460

3. Dussault J, Ruel J 1987 Thyroid hormones and brain development. Ann Rev Physiol 49:321-334

4. Rastogi RB, Lapierre Y, Singhal RH 1976 Evidence for the role of brain biogenic amines in depressed motor activity seen in chemically thyroidectomized rats. J Neurochem 26:443-449

5. Savard P, Merand Y, DiPaolo T, Dupont A 1983 Effects of thyroid state on serotonin, 5-hydroxyindoleacetic acid and substance $\mathrm{P}$ contents in discrete brain nuclei of adult rats. Neuroscience 10:1399-1404

6. Rastogi RB, Singhal RL 1978 The effect of thyroid hormone on serotonergic neurons: depletion of serotonin in discrete brain areas of developing hypothyroid rats. Naunyn Schmiedebergs Arch Pharmacol 304:9-13

7. Singhal RL, Rastogi RB, Hrdina PD 1975 Impaired brain amine metabolism in neonates during altered states of thyroid function. [Minireview] Life Sci 17:1617-1626

8. Rastogi RB, Singhal RL 1979 Effect of neonatal hypothyroidism and delayed 1-thiidothyronine treatment on behavioral activity and norepinephrine and dopamine biosynthesis in discrete regions of rat brain. Psychopharmacology (Berlin) 62:287-293

9. Ito JM, Valcana T, Timiras PS 1977 Effect of hypo- and hyperthyroidism on regional monoamine metabolism in the adult rat brain. Neuroendocrinology 24:55-64

10. Schuijers JA, Walker DW 1987 Effect of thyroidectomy on tissue catecholamine and dopamine $\beta$-hydroxylase content in fetal sheep. Proceedings of the Australian Physiological and Pharmacological Society 18:108P (abstr)

11. Fisher DA, Dussault JH, Sack J, Chopra IJ 1977 Ontogenesis of hypothalamicpituitary-thyroid function and metabolism in man, sheep and rat. In: Greep RO (ed) Recent Progress in Hormone Research, Vol 33. Academic Press, New York, pp 59-107

12. Fraser M, Liggins GC 1988 Thyroid hormone kinetics during late pregnancy in the ovine fetus. J Dev Physiol 16:461-471

13. Thorburn GC, Hopkins PS 1973 Foetal and neonatal physiology. Proceedings of the Sir Joseph Barcroft Centenary Symposium. Cambridge University Press, Cambridge, UK, pp 488-507

14. Richards GE, Gluckman PD, Mannelli SC 1987 Neurotransmitters and their metabolites in the brains of fetal and newborn lambs. Life Sci 41:1880-1884

15. Gluckman PD, Parsons Y 1983 Stereotaxic method and atlas for the ovine fetal forebrain. J Dev Physiol 5:101-123

16. Gunn TR, Butler J, Gluckman P 1986 Metabolic and hormone responses to cooling the fetal sheep in utero. J Dev Physiol 8:855-866

17. Zar JH 1984 Biostatistical Analysis. Prentice-Hall Inc, Englewood Cliff, NJ, p 157

18. Dobbing J 1974 The later development of the brain and its vulnerability. In: Davis JA, Dobbing J (eds) Scientific Foundations of Paediatrics. Heineman Medical Books, London 